\title{
Producción de bioetanol a partir del mucilago de café (Coffea arabica. L). CURC. UNAH. Comayagua. Honduras. 2011
}

Mario Renan Funes Caballero* Carlos Luis Banegas, Hamsy Maradiaga Gonzales, Melsar Enoc Osorio Rivera, Nelson Daniel Henríquez Flores, Néstor Eloy, Nicolás Mejía**

\section{RESUMEN}

La obtención de bioetanol a partir del mucilago de café, el cual es un producto de desecho que es arrojado a corrientes de agua, sin tratamiento alguno, ya que el productor no cuenta con alternativas de uso, causando graves problemas de contaminación. El estudio detalla acerca del aprovechamiento de mucilago generado durante el proceso de beneficiado del fruto, siendo los lugares de estudio La Libertad, en el Departamento de Comayagua y Marcala, en el Departamento de La Paz. Los dos métodos de fermentación utilizado son: en el método controlado se inocularon tres muestras, cada una con diferentes grados brix de 2000 mililitros de mucilago $+2 \%$ Peso/Peso, 2000 mililitros de mucilago+4\%Peso/Peso y 2000 mililitros de mucilago $+6 \%$ Peso/Peso de Saccharomyces cerevisae y el método no controlado sin inoculación de levadura, en el cual se utilizaron tres muestras de 2000 mililitros con diferentes grados Brix. Para cada uno de los métodos se destilaron 800 mililitros haciendo dos repeticiones de cada una de las muestras.

Los análisis realizados demuestran que existen factores que influyen para obtener rendimientos satisfactorios: la altura, concentración de azúcar, beneficiado del café y métodos de inoculación, entre otros. Con esto también se pretende sensibilizar a instituciones involucradas en la extensión rural y a los productores de café, en la implementación del bioetanol como fuente de energía alterna, como combustible de automóviles y otros usos de beneficio humano, y al mismo tiempo dar respuesta al problema ambiental que representan las aguas mieles de café (mucilago) en las distintas zonas de nuestro país.

Palabras Clave: Mucilago, bioetanol, aprovechamiento de residuos de café ABSTRACT

\footnotetext{
* Universidad Nacional Autónoma de Honduras. Centro Universitario Regional de Comayagua (CURC). Ingeniero Agrónomo. funescaballero@yahoo.com

** Universidad Nacional Autónoma de Honduras. Centro Universitario Regional de Comayagua (CURC). Ingenieros Agroindustriales. www.wix.com/curcunah/cure
} 
Getting ethanol from coffee mucilage, which is a waste product that is thrown into running water, without treatment, because the producer has no alternative use, causing serious pollution problems. The study details about the use of mucilage produced during the milling process of the fruit, being the study sites in La Libertad, Comayagua Department and Marcala, La Paz Department. The two fermentation methods used are: in the controlled method were inoculated three samples, each with different brix degrees of 2000 milliliters of mucilage $+2 \%$ Weight / Weight, 2000 $\mathrm{ml}$ of mucilage $+4 \%$ weight / weight and $2000 \mathrm{ml}$ of mucilage $+6 \%$ Weight / Weight of Saccharomyces cerevisiae and the method controlled without inoculation of yeast, which were used in three samples of 2000 milliliters with different Brix degrees. For each of the methods 800 milliliters were distilled doing two repetitions of each of the samples.

The analysis shows that there are factors that influence to obtain satisfactory yields: height, concentration of sugar, coffee processing and inoculation methods, among others. This also aims to raise institutions involved in rural extension and coffee producers in the implementation of bioethanol as alternative energy source as fuel for cars and other uses of human benefit, while responding to the environmental problem representing the wastewater of coffee (mucilage) in different parts of our country.

Key words: Mucilage, bioethanol, use of cofee's waste. 


\section{INTRODUCCIÓN}

El mucílago de café está constituido por el mesocarpio del fruto y representa el 15$20 \%$ del peso del grano fresco; el café uva maduro presenta una composición física tal que los granos representan el $20 \%$ del fruto, el proceso de despulpado genera un $80 \%$ de rechazo que es tirado por los caficultores a las quebradas y pequeños riachuelos, todos los años, generando contaminación a las fuentes y la desaparición de especies acuáticas. El mucilago de café se puede utilizar para producir bioetanol, es un medio completo conteniendo sus características elementales. El principio activo del mucilago del café son los grados Brix, que son los que actúan en el proceso de fermentación en el cual los carbohidratos son transformados en azúcares simples por medio de la levadura del genero Saccharomyces, que lo convierten en etanol pasando luego por el proceso de destilado y así obtener bioetanol como producto final.

La investigación es la contribución en la producción de biocombustible, obtenida a partir de mucilago de café, debido a que existe una debilidad respecto a la falta de investigación en esta área. Estos resultados podrán aplicarse al producto de desecho de café en las diferentes zonas de nuestro país. Como resultado de este estudio se ha comprobado que la altitud de los lugares es un potencial para producir bioetanol.

Si bien es cierto que la producción de bioetanol en nuestro país apenas comienza a dar frutos, se puede empezar a implementar los conocimientos adquiridos y desarrollar tecnologías que puedan hacer de nuestro país más competitivo y contribuir de esta manera a la conservación del medio ambiente, dando alternativas que se manifiesten en el desarrollo, así como la generación de biocombustibles que puedan de alguna manera ser una alternativa de combustibles fósiles. La producción de bioetanol beneficiará a todos los productores de las zonas cafetaleras ya que en el proceso de beneficiado del café, los residuos no están siendo aprovechados, especialmente el mucilago, siendo este un factor de alto grado de contaminación al medio ambiente de nuestro país.

\section{OBJETIVOS}

Evaluar el desempeño y productividad del proceso de fermentación anaeróbica más efectiva para la obtención de bioetanol, utilizando el método controlado y el no controlado, y los factores que influyen en el rendimiento y la reducción de la contaminación mediante la utilización adecuada del mucilago de café. 


\section{MATERIALES Y MÉTODOS}

\section{Obtención de la materia prima}

El mucílago fresco fue recolectado en cuatro beneficios: La Soledad, ubicado en la aldea Nueva Esperanza, Hernández, aldea Valle Bonito y La Cidra, aldea La Cidra; los beneficios pertenecen a La Libertad, Comayagua, Honduras. Las zonas cafetaleras están ubicadas a una altitud que oscila entre los 800 a $1500 \mathrm{msnm}$. El cuatro beneficio Sogimex que se encuentra ubicado en Choacapa, Chinacla, La Paz, Honduras. La zona se encuentra a una altitud de 1250 a 1300 msnm.

Las muestras en los beneficios se obtuvieron al inicio de la cosecha. El cual difiere en el momento de mayor volumen de cosecha y a la madurez del fruto. Mientras mayor sea la altitud en la que se encuentre sembrado el café, mayor es el contenido de azúcares en el fruto del café y por ende mayor es la cantidad de mucilago presente en el fruto.

\section{Diseño Experimental}

El estudio fue establecido en el laboratorio de Ingeniería agroindustrial. CURC. UNAH, mediante un diseño de bloques completamente al azar (BCA) con dos repeticiones. Los datos recolectados para las distintas variables fueron sometidos a un análisis de varianza (ANAVA, $p<0.05$ ) utilizando el paquete estadístico Infostat versión 2010 de la Universidad de Córdoba. Argentina.

\section{Variables a evaluar}

Porcentaje y densidad del etanol en los procesos de fermentación controlada y no controlada. Los promedios fueron sometidos a una prueba de Duncan, la cual nos permite definir si las diferencias son significativas o no $(p<0,05)$. Se empleó el programa MS Excel 2010, para realizar graficas.

\section{Para el proceso de fermentación controlada}

Se utilizaron dos litros de mucílago fresco diluido por cada tratamiento, sumando un total de seis litros de mucílago del beneficio Sogimex. En el proceso de pasteurización se inoculó con un $2 \%, 4 \%$, y 6\% de levadura Saccharomyces cerevisae. Se presenta el siguiente diagrama: 
Figura 1. Diagrama de flujo del proceso de fermentación, método controlado. Laboratorio de Ingeniería Agroindustrial. CURC. UNAH. Comayagua. Honduras. 2011

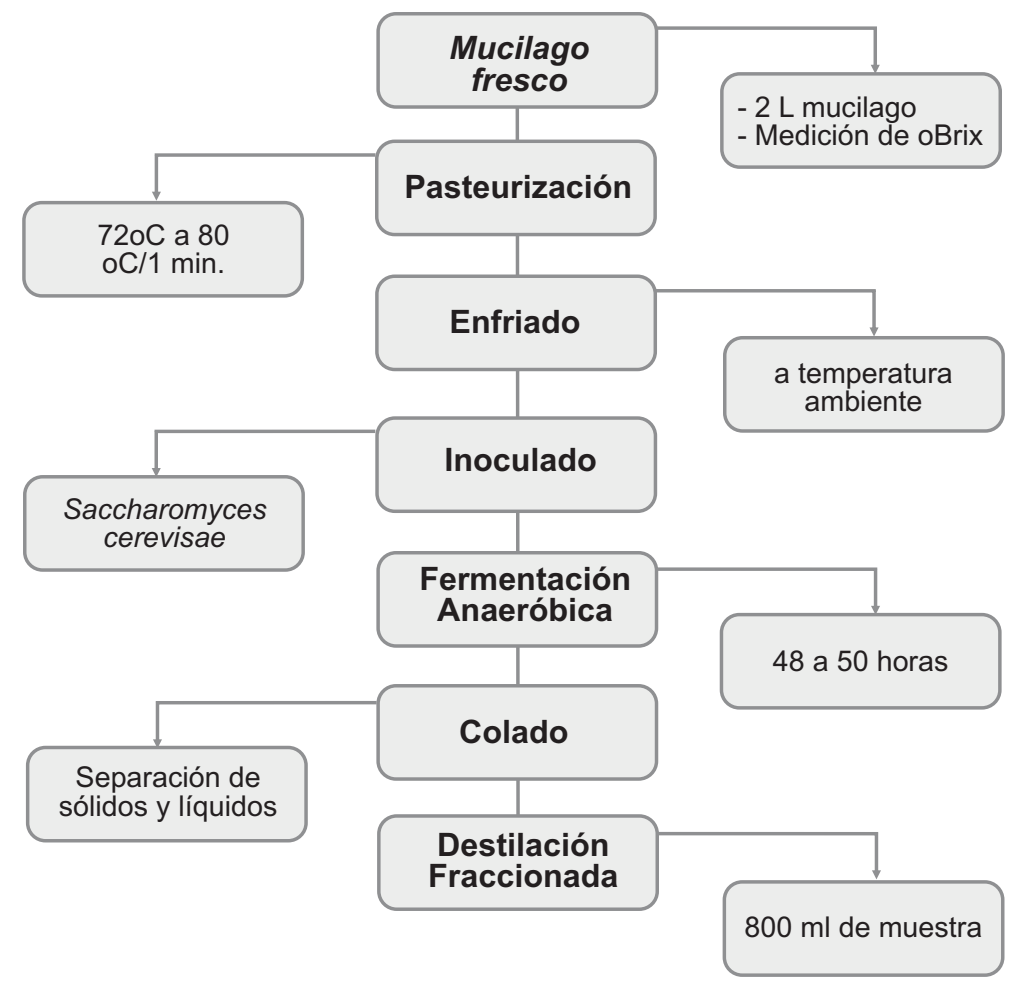

\section{Fermentación anaeróbica controlada}

La fermentación es un proceso en el cual se llevan a cabo cambios químicos en un substrato orgánico, ya sea en hidrato de carbono, proteína, o grasa. Estos cambios se llevan a cabo por catalizadores bioquímicos conocidos como enzimas, que son elaboradas por tipos específicos de microorganismos vivos. El método de fermentación anaeróbica controlada consiste en primera instancia del pesado inicial del producto, para luego llevar a cabo el proceso de pasteurización de $72 \mathrm{a} 80^{\circ} \mathrm{C}$ por un periodo de un minuto, para eliminar microorganismos antagónicos, posteriormente se deja enfriar el producto a temperatura ambiente y una vez efectuado esto, se inocula con levaduras Saccharomyces cerevisae previamente activadas para la preparación del inoculo; se pesa la levadura en 2, 4 y $6 \%$ Peso/Peso por separado, luego se disuelve en agua tibia y se le adiciona inmediatamente al mucílago pasteurizado. Finalmente, se deja el mucílago en 
proceso de fermentación anaeróbica. Para la destilación fraccionada se toman 800 mililitros de muestra previamente colada, el proceso de colado del producto fermentado reduce el tiempo de volatilización del etanol al momento de la destilación debido a que en ella no hay presencia de sólidos pesados, los cuales dificultan el proceso de evaporación. En la pasteurización no se deben rebasar los $80^{\circ} \mathrm{C}$, con el fin de evitar que el producto se caramelice por efecto del contenido de azúcares bajo un proceso de alta temperatura; es necesario mantener el rango de temperatura para eliminar la mayor cantidad posible de microorganismos. Una vez enfriado el producto, la levadura se debe activar con agua tibia y posteriormente diluirla en el mucílago previamente pasteurizado. Es importante monitorear frecuentemente el tiempo de fermentación, ya que ésta puede ser lenta o rápida, dependiendo de la agilización de los agentes descomponedores y del medio ácido en que se encuentren.

\section{Proceso de fermentación no controlado}

Se utilizaron dos litros de mucílago de cada beneficio de la zona de La Libertad, Comayagua, sumando 6 litros de mucílago en total. Para este método no se empleó el proceso de pasteurización, ni de inoculación, siendo así un proceso de fermentación natural, mismo que se administró a partir del diagrama de flujo presentado en la Figura 2. Una vez fermentado el producto (esto puede variar de acuerdo a la zona o a la diferencia de tratamientos respecto al contenido de grado Brix del mucílago), se realizó la destilación fraccionada en el laboratorio del Centro Universitario Regional del Centro (CURC). Por cada tratamiento se utilizaron 800 mililitros de producto fermentado para la destilación, con 2 repeticiones por cada tratamiento. Este principio de destilación fraccionada aplica tanto para el método de fermentación anaeróbica controlada como la de fermentación no controlada.

Figura 2. Diagrama de flujo del proceso de fermentación, método no controlado. CURC. UNAH. Comayagua. Honduras. 2011

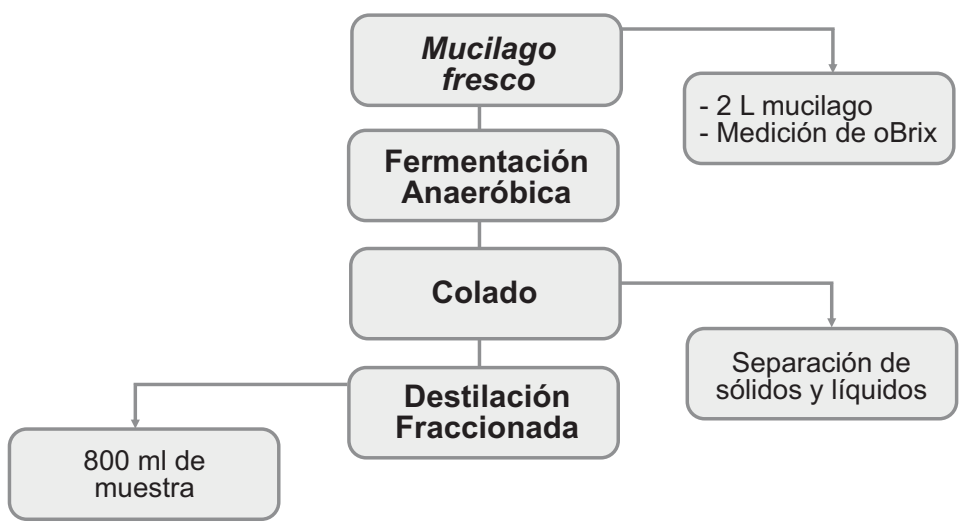

Dirección de Investigación Científica $\longdiv { 1 5 5 }$ 


\section{Fermentación anaeróbica no controlada}

En el método no controlado, se midieron los grados Brix del mucilago, se deja fermentar el producto en ausencia de oxígeno sin pasteurizar. El tiempo de fermentación varía dependiendo de los grados Brix del mucilago. Una vez logrado esto, se realiza la destilación fraccionada tomando 2 repeticiones de 800 mililitros de muestra para cada beneficio, debe estar previamente colada.

Es importante considerar que el mucílago a recolectar debe ser fresco y con mayor contenido de azúcares. En el método no controlado puede variar, pudiendo ser más lento por no estar inoculado con levaduras en su proceso de fermentación. Si no se observa la salida de $\mathrm{CO}_{2}$ en el proceso de fermentación, el producto está listo para destilar. En el principio de la fermentación bajo condiciones anaeróbicas, la glucosa es convertida en etanol y dióxido de carbono $\left(\mathrm{CO}_{2}\right)$ mediante el fenómeno denominado glucólisis en el ciclo de fermentación.

\section{Proceso de destilación fraccionada}

Después de haber fermentado el producto con el método controlado y no controlado (natural) y previamente colado, se procedió a realizar la destilación fraccionada.

a. Antes de llevar el producto fermentado en el balón, se realizó una separación de sólidos y líquidos por medio de un colado. El producto a fermentar en líquido será más fácil de evaporarse al momento de la destilación, sin la presencia de sólidos pesados.

b. Se colocó una primera muestra de 800 mililitros de producto fermentado en un balón de destilación.

c. Se calentó muy suavemente el contenido del balón de destilación y se mantuvo así hasta que llegó a los $75^{\circ} \mathrm{C}$, sin dejar que pasara de los $80^{\circ} \mathrm{C}$.

d. Posteriormente se recolectó el líquido destilado entre $75 \mathrm{a} 80^{\circ} \mathrm{C}$.

e. Se repitieron los pasos anteriores para las demás muestras a destilar, tomando nuevamente 800 mililitros de muestra, logrando así las dos repeticiones por cada tratamiento.

\section{RESULTADOS Y DISCUSIÓN}

\section{Destilación fraccionada del método controlado}

En el cuadro 1 se muestran las variables que son efecto de las diferentes concentraciones de Saccharomyces Cerevisae de donde se obtuvieron los 
siguientes resultados:

Cuadro 1. Densidad, porcentaje de etanol, brix y volumen de etanol. Resultado de método y mediciones controladas. CURC. UNAH. Comayagua.

Honduras. 2011.

\begin{tabular}{|c|c|c|c|c|}
\hline $\begin{array}{c}\text { Muestras } \\
\text { (Tratamientos) }\end{array}$ & Densidad (gr/ml) & $\%$ de etanol & Brix & Vol / etanol (ml) \\
\hline $\begin{array}{c}800 \mathrm{ml} \text { de } \\
\text { mucilago con 6\% } \\
\text { de levadura }\end{array}$ & $0.857 \mathrm{a}$ & $0.175 \mathrm{a}$ & 6.0 & 1.4 \\
\hline $\begin{array}{c}800 \mathrm{ml} \text { de } \\
\text { mucilago con } 4 \% \\
\text { de levadura }\end{array}$ & $0.811 \mathrm{ab}$ & $0.075 \mathrm{~b}$ & 5.0 & 0.6 \\
\hline $\begin{array}{c}800 \mathrm{ml} \text { de } \\
\text { mucilago con } 2 \% \\
\text { de levadura }\end{array}$ & $0.751 \mathrm{~b}$ & $0.10 \mathrm{~b}$ & 5.5 & 0.8 \\
\hline
\end{tabular}

\section{Análisis de la densidad del alcohol etílico obtenido}

En la gráfica 1 se observan los resultados de los tres tratamientos inoculados con levadura Saccharomyces cerevisae.

Con 800 mililitros de mucilago inoculado con levadura al $6 \%$ Peso/Peso se obtuvo un leve aumento, con respecto a la densidad del etanol de $0.857 \mathrm{gr} / \mathrm{ml}$, en comparación con 800 mililitros de mucilago inoculado con levadura al 4\%Peso/Peso, que fue de $0.811 \mathrm{gr} / \mathrm{ml}$.

Sin embargo, con 800 mililitros de mucilago inoculado con levadura al $2 \%$ Peso/Peso la densidad fue de $0.751 \mathrm{gr} / \mathrm{ml}$, siendo el tratamiento inoculado con levadura al $4 \%$ Peso/Peso el que más se acercó a la densidad real del etanol, siendo ésta de $0,795 \mathrm{gr} / \mathrm{ml}$.

Finalmente, entre las medias de la densidad de etanol obtenido hay diferencia significativa a que los tres tratamientos no presentan un mismo comportamiento, según los rangos múltiples de la prueba de Duncan. 
Gráfico 1. Densidad del etanol de tres diferentes concentraciones de levadura con respecto a peso/peso.CURC-UNAH. Comayagua. Honduras. 2011

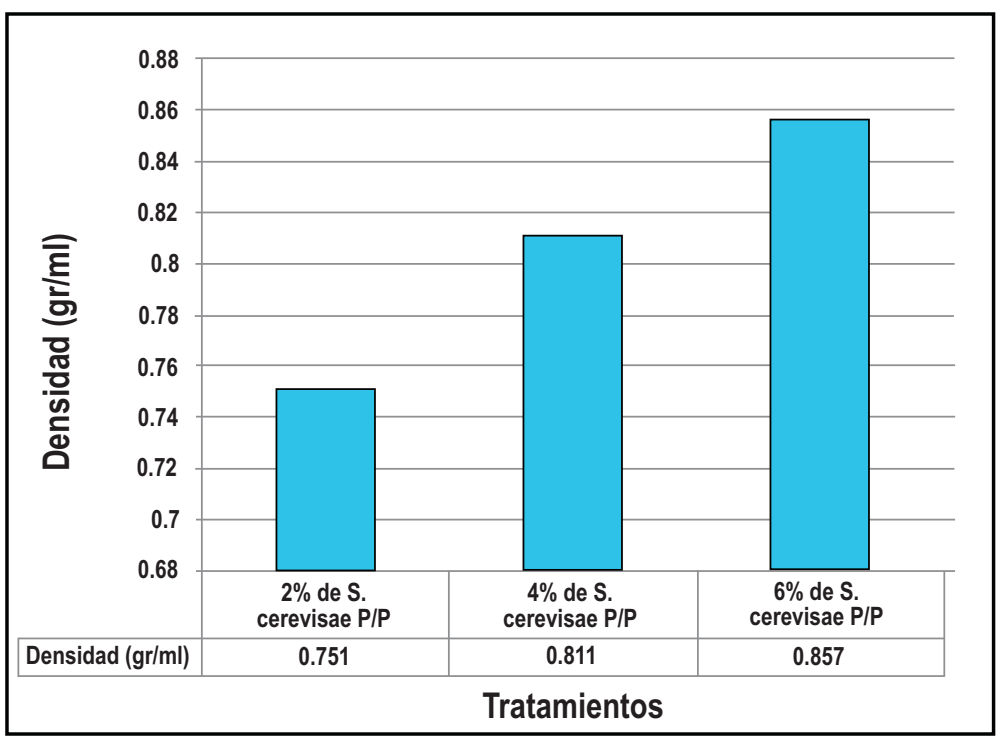

El resultado de las densidades altas en cada una de las medias de los tratamientos con respecto a la densidad real del etanol difiere significativamente Como lo demuestran estos análisis, debido a que el mucilago fresco estaba diluido con agua y esto provocó que el contenido de azúcares disminuya. Por haber poca glucosa, el proceso del fermentado por medio de la glucólisis produce poco alcohol. Al momento de realizar la destilación fraccionada se comienza a evaporar agua y se volatiliza el etanol, y éstos al mezclarse comienzan a equilibrar sus densidades del agua. Los mejores resultados se obtienen cuando se utiliza mucilago fresco y puro, sin diluir.

\section{Análisis del porcentaje de etanol obtenido}

En el gráfico 2 " puede observar que en el tratamiento con 6\%Peso/Peso de levadura se obtuvo $0.175 \%$ de etanol, mientras que en el tratamiento de $2 \%$ Peso/Peso de levadura el porcentaje fue $0.1 \%$ y en el tratamiento de $4 \%$ Peso/Peso de levadura se obtuvo un porcentaje de $0.075 \%$ a partir de 800 mililitros de mucilago destilados para cada uno de los tratamientos. Esto significa que de 800 mililitros de muestra destilada se obtiene un rango de 1.4 a 0.8 mililitros de alcohol etílico, lo cual traducido a 1,000 litros de mucílago diluido se obtendrían en promedio 1.166 litros de etanol. Sin embargo, si el mucilago es fresco y puro, sin diluir, el resultado de obtener alcohol etílico sería siete veces mayor. 
Gráfico 2. Porcentaje de etanol obtenido en 800 mililitros de muestra destilada. CURC. UNAH. Comayagua. Honduras. 2011

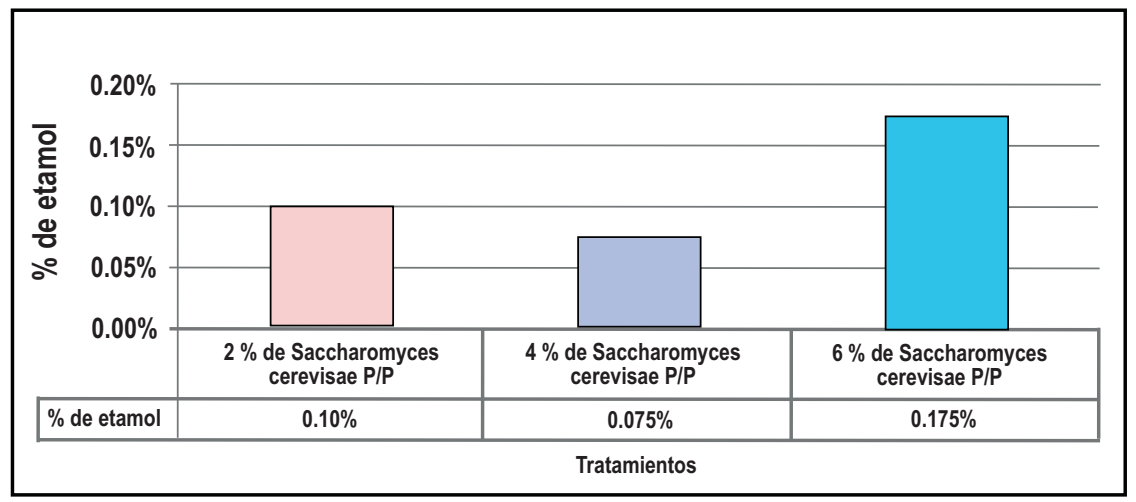

\section{Relación de grados Brix con el porcentaje de etanol obtenido. Método controlado}

En el gráfico 3 se observa que por cada 2 grados Brix se obtiene un $1 \%$ de alcohol etílico. En el tratamiento con $6 \%$ Peso/Peso de levadura encontramos que el mucílago contiene 6.0 grados Brix y que nuestra producción de etanol sería técnicamente de $3.0 \%$. Por otro lado, para el tratamiento con $2 \%$ Peso/Peso de levadura, que presenta 5.5 grados brix, a producción de etanol sería de $2.75 \%$, y para el tratamiento con 4\%Peso/Peso la llevadura tiene 5.0 grados brix y la producción de etanol sería de $2.5 \%$. Los tres tratamientos con levadura fueron inoculados en 2 litros de mucilago fresco diluido.

Gráfico 3. Efecto de grados Brix del mucilago fresco con respecto al porcentaje de etanol obtenido en 800 mililitros de muestra. Método Controlado. CURC.

UNAH. Comayagua. Honduras. 2011

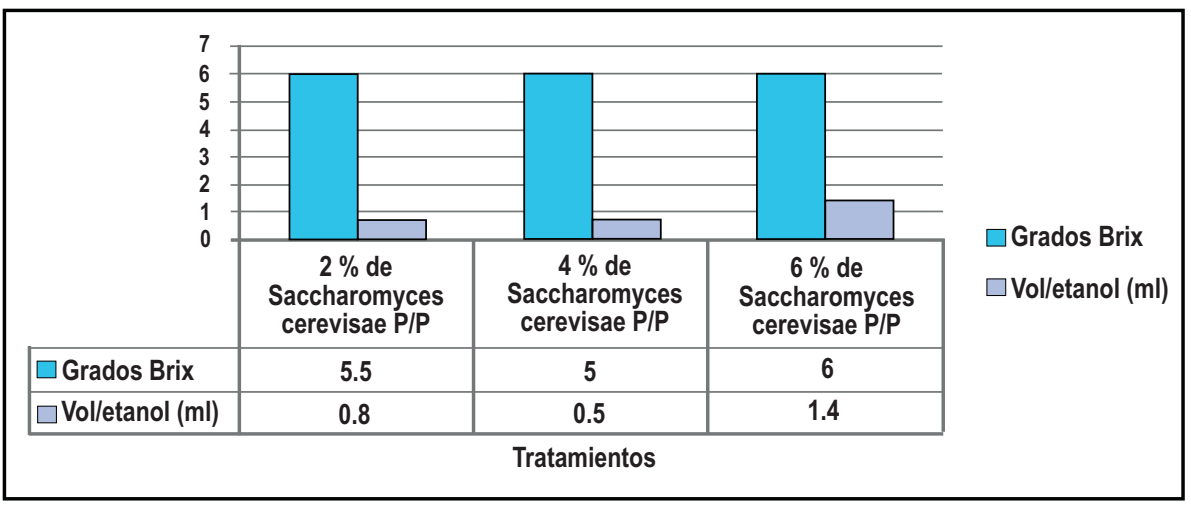


El beneficio Sogimex, que se encuentra a una altitud que oscila entre 1924 a 2006 msnm y cuya relación de grados Brix de las muestras son: Para el tratamiento con $6 \%$ Peso/Peso de levadura tenía 6.0 grados Brix, mientras que el tratamiento con $2 \% \mathrm{Pes} / \mathrm{Peso}$ de levadura tenía 5.5 grados Brix y el tratamiento con 4\%Peso/Peso de levadura tenía 5.0 grados Brix, siendo estas cantidades de grados Brix no recomendables para la producción de bioetanol ya que tiene que ser mayor de 8 grados Brix para obtener mejores rendimientos en la producción. Otra variable importante que determina el porcentaje de etanol es qué tan diluido se encuentre el mucilago, ya que al momento del proceso de despulpado el mucilago tiende a mezclarse con el agua, lo que ocasiona que la concentración de grados Brix del mucilago disminuya.

\section{Destilación fraccionada del método no controlado}

En el cuadro 2 se representan los mismos parámetros aplicados en el método controlado, únicamente diferenciado por las inoculaciones y pasteurización no aplicada a estas muestras. Y se detalla las variables a discutir que son de los tres diferentes Beneficios: Beneficio La Soledad, Beneficio Hernández y Beneficio La Cidra, ubicados en la zona de La Libertad, Comayagua en donde se calcularon cada uno de estos por separado.

\section{Análisis de la densidad del alcohol etílico obtenido}

En el gráfico 4 se muestran los resultados de las densidades de los tres diferentes beneficios, siendo la densidad del Beneficio Hernández de 0,813gr/ml mientras que la del Beneficio La Cidra es 0,789gr/ml y la del Beneficio La Soledad de 0,712gr/ml, siendo la muestra del Beneficio La Cidra la que más se acercó a la densidad real del etanol que es de $0,795 \mathrm{gr} / \mathrm{ml}$.

Gráfico 4. Densidad del etanol de tres diferentes Beneficios: La Soledad, Hernández y La Cidra. CURC. UNAH. Comayagua. Honduras. 2011

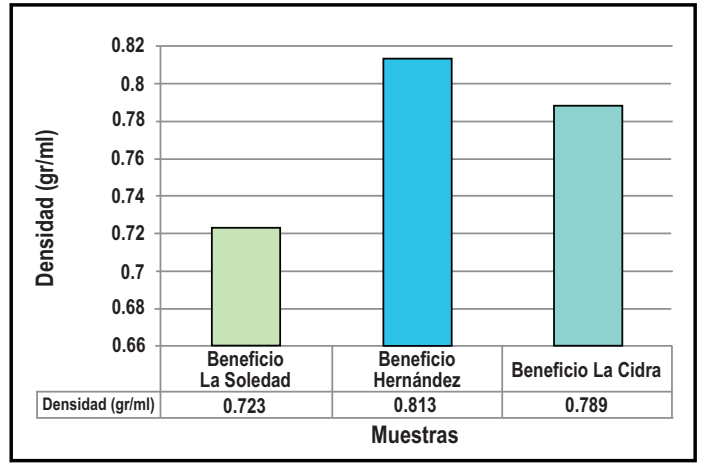




\section{Análisis del porcentaje de etanol obtenido}

Los porcentajes de alcohol etílico obtenidos en los tres diferentes Beneficios son: Beneficio La Cidra $0.106 \%$, mientras que en el Beneficio La Soledad es $0.075 \%$ y en el Beneficio Hernández es de $0.063 \%$. En este tratamiento no se utilizaron cultivos de Saccharomyces cerevisae. (Gráfico 5).

Gráfico 5. Porcentaje de etanol obtenido en 800 mililitros de muestra destilada.

CURC. UNAH. Comayagua. Honduras. 2011

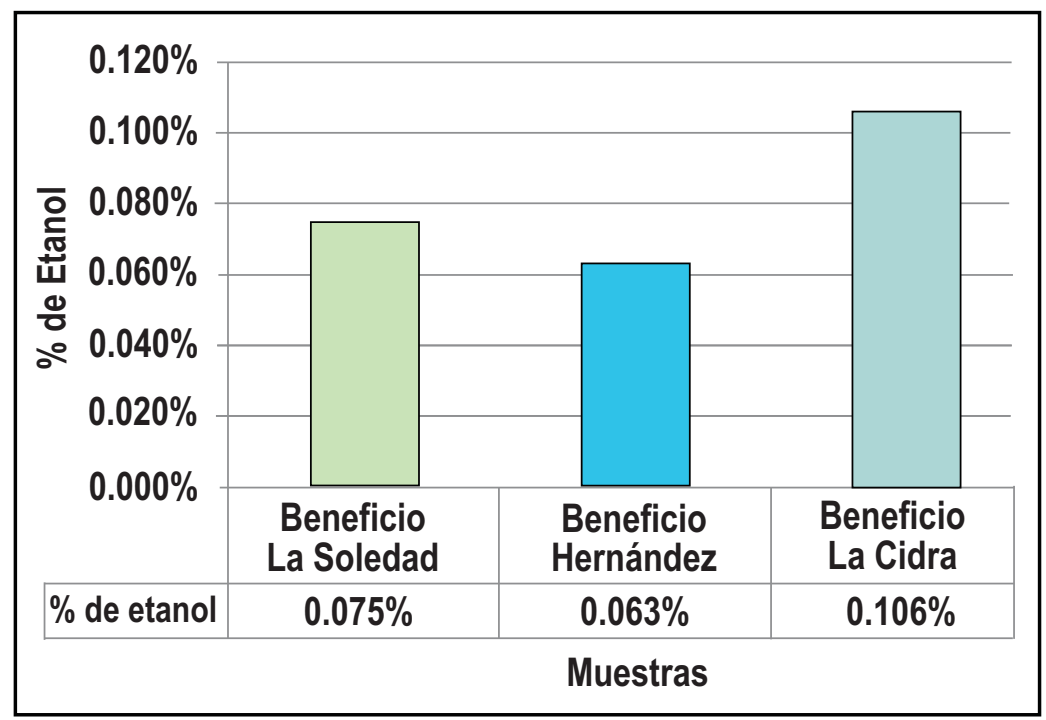

Relación de grados Brix con el porcentaje de etanol obtenido. Método no controlado

Según el gráfico 6, por cada 2 grados Brix se obtiene un $1 \%$ de alcohol etílico. En la muestra del Beneficio La Soledad se encontró que el mucilago contenía 4.0 grados Brix y que nuestra producción de etanol sería técnicamente de $2.0 \%$.

Por otro lado, para el caso del Beneficio Hernández, que presenta 3.8 grados Brix, la producción de etanol sería de $1.9 \%$, y para el caso del Beneficio La Cidra, que tiene 4.5 grados Brix, la producción de etanol sería de $2.25 \%$. 
Gráfico 6. Efecto de grados Brix del mucilago fresco con respecto al porcentaje de etanol obtenido en 800 mililitros de muestra, método no controlado.

CURC. UNAH. Comayagua. Honduras. 2011.

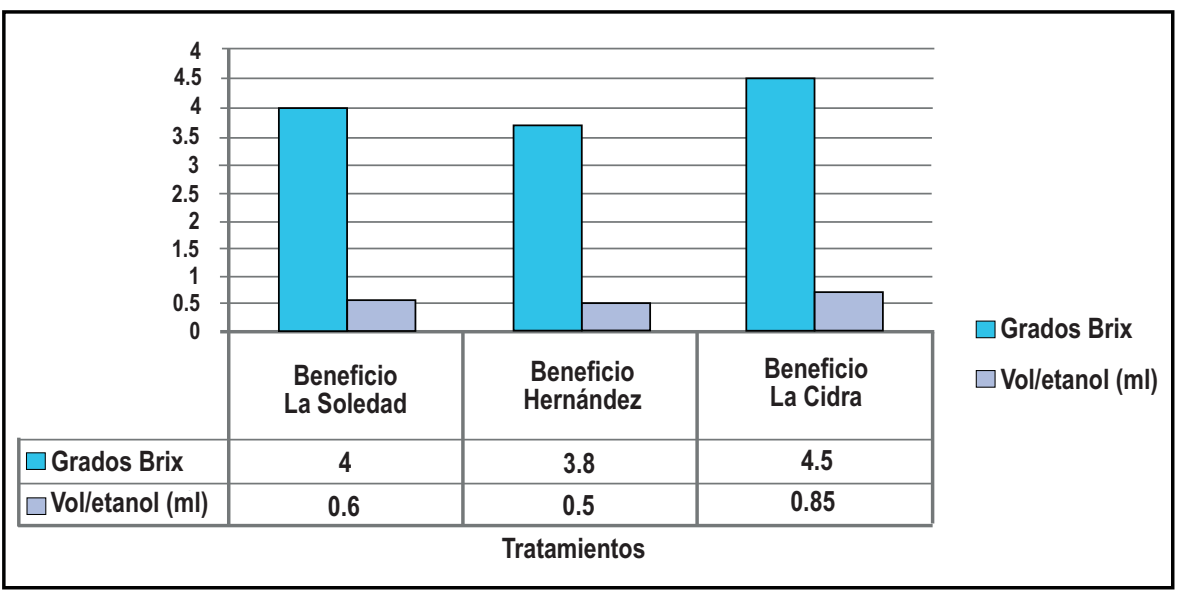

Los lugares en estudio presentan las siguientes producciones: en la zona de La Libertad se producen 110,852 quintales oro por el periodo y, conociendo datos, para obtener un quintal oro se necesitan 5.7 quintales de café uva. En las zonas en estudio se han implementado los beneficios ecológicos, dicha clasificación se basa en el consumo de agua que cada uno de estos gasta y dentro del intervalo conocido se maneja 180-250 litros de agua consumida por qq oro (IHCAFE 2011). Tomando como punto de partida esta información se calcula que la materia prima disponible para tal situación planteada será: 110,852 qq oro X 250 litros de agua $=27,713,000$ litros de agua con mucílago. De las cuales, según los datos del IHCAFE, el $60 \%$ del rechazo representa mucílago y el otro $40 \%$ es pulpa, con lo que la materia prima disponible sería: $27,713,000$ litros de agua con mucílago $X 60 \%=16,627,800$ litros de mucílago. Se obtendrían 20,784.75 litros de etanol (5,530.8 galones) por temporada ya que de cada 1000 litros de mucílago se obtendrían 1.25 litros de etanol.

En la zona de Macala se producen 54,912.28 qq oro durante la temporada y tomando en cuenta la relación arriba descrita de café uva y oro, por lo tanto se tiene que: $54,912.28$ qq oro $X 250$ litros de agua $=13,728,070$ litros de agua con mucilago. $Y$ de acuerdo a los datos proporcionados por el incafe en relación al porcentaje de mucilago comprendido en las aguas mieles de detalla: 13,728,070 Itros de agua con mucilago X $60 \%=8,236,842$ litros de mucilago. Por lo que el volumen de etanol producido durante la temporada constaría de 10,296.05 litros (2,739.8 galones). 


\section{CONCLUSIONES}

- Es posible obtener bioetanol en proporciones mayores si se cuenta con un equipo que requiera menos agua en el despulpado del café para recolectar mucílago más puro.

- Los productores no se ven interesados en implementar esta clase de tecnologías, ya que no cuentan con los medios suficientes y les es más factible producir abonos orgánicos del mucílago y otros subproductos del café.

- Si se llegara a producir bioetanol, el método más factible para la obtención es utilizando el método de fermentación controlado inoculado con Saccharomyces cerevisaeal $2 \%, 4 \%$ y $6 \%$.

- La altura de las zonas y el tiempo de recolección de las muestras influyen en la producción de bioetanol, ya que a mayor altura sobre el nivel del mar y en plena cosecha del café, mejor será la materia prima y la concentración de azúcares que ayudarán a la fermentación.

- Es importante recordar que el bioetanol emite igual (o muy similar) cantidad de $\mathrm{CO}_{2}$ que los combustibles fósiles y si bien uno de sus beneficios es ser un recurso renovable, el verdadero incentivo en su uso está en el balance global de $\mathrm{CO}_{2}$, pues las plantas necesarias para producir bioetanol consumen más $\mathrm{CO}_{2}$ del que emite el bioetanol producido. Desde el punto de vista energético aun se debate si su balance es favorable o desfavorable.

- El proceso resulta barato dado que la materia prima no tiene hasta ahora un valor comercial (producto de desecho) y los insumos son de fácil acceso, por lo que resultaría atractivo a la industria comparado con el etanol de caña de azúcar.

- El control de temperatura influye gradualmente en el proceso de la destilación fraccionada, ya que a temperaturas inferiores a $70{ }^{\circ} \mathrm{C}$ se destila metanol, y por arriba de los $70^{\circ} \mathrm{C}$ se destila etanol, considerando que la temperatura de ebullición del etanol es de $78^{\circ} \mathrm{C}$.

\section{RECOMENDACIONES}

- Es necesario dar mayor importancia a los procesos investigativos, facilitando laboratorios y asignando personal encargado de llevar a cabo análisis que se requieran en cualquier estudio. Así mismo contar con los instrumentos, equipo, material e instalaciones necesarios para desarrollar trabajos eficientes con resultados más claros.

- Se recomienda hacer destilaciones para la obtención de bioetanol con mucílago inoculado ya que éste dará mejores resultados.

- Es importante tomar en cuenta la época de recolección del mucílago pues éste es 
mejor cuando el grano está en óptimo estado de maduración, por lo que se recomiendan los meses de noviembre a enero.

- Se recomienda seguir realizando futuras investigaciones con respecto a las diferentes materias vegetales de desecho que tengan potencialidad para producir los alcoholes y reducir los contaminantes del planeta.

- Para lograr una producción a nivel industrial se deberían construir plantas pilotos en diferentes zonas en donde existan asociaciones de productores o beneficios grandes, para obtener mucho mucilago y así obtener etanol.

\section{BIBLIOGRAFÍA}

ADECAFEH (Asociación de Exportadores de Café de Honduras). 2005. Origen del café (en línea). Tegucigalpa, HN. Consultado el: 4 de septiembre del 2011. Disponible en http://www.mayanet.hn/adecafeh/elgrano.htm

Chan, Y. 2006. Industrialización de alimentos, frutas y vegetales. Guácimo, CR, en línea. http://www.Universidad EARTH.com

Fessenden, R. J.; Fessenden, J. S. 1982. Química orgánica, 2. ed. México, D.F. Iberoamericana. $1075 \mathrm{p}$.

IHCAFE. (Instituto Hondureño del Café). 2001. Manual de Caficultura. Instituto Hondureño del café. Tegucigalpa. M.D.C. 203 p.

INFOCOOP (Instituto Nacional de Fomento Cooperativo, CR). 2006. Café Cooperativa. Palmares. Consultado el: 26 de septiembre del 2011. Disponible en:

http://infocoop.bankoi.com/costaricacoop/tev/estandar/pag.php?id=130

Pérez Fonseca, K.; Molina Villalobos, K. 2005. Una alternativa de desarrollo local sostenible: Asociación Agroecoturística de la Argentina de Pocora, Limón, Costa Rica. In Congreso Internacional de Casos Exitosos de Desarrollo Sostenible del Trópico (1, 2005, Veracruz, MX). [Memorias] (en línea). Veracruz, MX, Universidad de Veracruz. Consultado el 26 de septiembre del 2011. Disponible en

http://www.uv.mx/CITRO/reunion/acrobat/E1PDF13.pdf\#search= $\% 22 \% 22$ Una\%20alternativa $\% 20$ de $\% 20$ desarrollo $\% 20$ local $\% 20$ sostenible $\% 22 \% 22$

Producción de bioetanol, biogás y biofertilizantes a partir de los desechos del beneficiado del café en Marcala. La experiencia de COMSA Honduras, Consultado 26 sep. 2011. Disponible en:

http://www.snvla.org/mm/file/HN\%20SNV05\%20\%20Bioetanol\%20v7\%20 Centroamerica.pdf 